\title{
Phase diagram of MgO from density-functional theory and molecular-dynamics simulations
}

\author{
Alejandro Strachan, Tahir Çağin, and William A. Goddard III \\ Materials and Process Simulation Center, Beckman Institute (139-74), California Institute of Technology, Pasadena, California 91125
}

(Received 13 May 1999)

\begin{abstract}
We use first-principles methods (no empirical parameters) to establish the phase diagram for the $\mathrm{B} 1(\mathrm{NaCl})$, $\mathrm{B} 2(\mathrm{CsCl})$, and liquid phases of $\mathrm{MgO}$. We used density-functional theory with the generalized gradient approximation to predict the equation-of-state [volume versus pressure $(V(P))$ ] at $0 \mathrm{~K}$ for $\mathrm{MgO}$ in the low-density $\mathrm{B} 1$ $(\mathrm{NaCl})$ phase and the high-density $\mathrm{B} 2(\mathrm{CsCl})$ phase. We find a pressure-induced phase transition at $P=400$ $\mathrm{GPa}$. We then fitted an MS-Q type force field (FF) to the quantum results. This FF, denoted as qMS-Q FF, was then used in molecular dynamics (MD) simulations to investigate the phase coexistence curves of the B1-B2 and B1-liquid phases. This leads to a first-principles phase diagram for $\mathrm{MgO}$ for pressures up to $500 \mathrm{GPa}$ and temperatures up to $8000 \mathrm{~K}$. The accuracy of the fit of the qMS-Q FF to the quantum mechanics validates the functional form of the qMS-Q FF in which the charges are obtained from charge equilibration (QEq) and the nonelectrostatic forces are described with simple two-body Morse potentials. Such qMS-Q FF using no empirical data should be useful for MD or Monte Carlo simulations of many other materials.

[S0163-1829(99)02346-2]
\end{abstract}

\section{INTRODUCTION}

Magnesium oxide, one of the most abundant oxides in the earth's lower mantle, has been studied extensively, both experimentally and theoretically. It has become a prototype for testing our ability to understand the physics of materials at ultrahigh pressures. The equation-of-state (EOS) (the relation between $P, V$, and $T$ ) is the most fundamental property obtained from these studies. Despite numerous studies of $\mathrm{MgO}$ at high pressures and temperatures, there remain significant questions about its phase diagram. Most notably the pressure at which the B1 (sodium chloride, six coordinated) structure transforms to the B2 (cesium chloride, eight coordinated) structure is unknown. Experiment shows the B1 structure to be stable for pressures up to $227 \mathrm{GPa}^{1}$ On the other hand, various quantum mechanics (QM) studies $^{2-6}$ predict the pressure for the B1-B2 phase transition at $T=0 \mathrm{~K}$ to be as low as $205 \mathrm{GPa}$ (Ref. 3) and as high as $1050 \mathrm{GPa}^{2}$ The most reliable of these calculations ${ }^{6}$ gives $450 \mathrm{GPa}$.

There is also uncertainty in the melting point of $\mathrm{MgO}$. Thus, at $P=25 \mathrm{GPa}$ recent simulations ${ }^{7-9}$ lead to $T_{\mathrm{MP}}$ $\sim 5000 \mathrm{~K}$, whereas experiment leads to $T \sim 3700 \mathrm{~K} .{ }^{10}$ Cohen and Gong ${ }^{11}$ studied melting in $\mathrm{MgO}$ clusters and found higher melting temperatures than the ones in the simulations mentioned above. However, the theoretical and experimental methods both have significant uncertainties. The simulations usually treat the solid as an infinite periodic system, and hence lack the surface sites that normally nucleate a new phase. In addition, by starting with a perfect crystal at low temperature and heating for relatively short times $(\sim \mathrm{ns})$ the cell might not attain the equilibrium density of defects in solid phase. Both effects would tend to yield $T_{\mathrm{MP}}$ too high and to a broadening of the phase transition. ${ }^{12}$ On the other hand, the experiments involve nonhydrostatic stresses and impurities which might decrease the observed $T_{\mathrm{MP}}$ below the intrinsic values at high pressures. ${ }^{1}$

Herein we use accurate QM [density-functional theory using the generalized gradient approximation (DFT-GGA)] at $0 \mathrm{~K}$ to develop a force field $(\mathrm{FF})$ accurate for pressures up to $500 \mathrm{GPa}$ and then use this $\mathrm{FF}$ in molecular-dynamics (MD) calculations to study the EOS for the B1, B2, and liquid phases of $\mathrm{MgO}$ for $T$ up to $8000 \mathrm{~K}$. The zerotemperature EOS for the $\mathrm{B} 1$ and $\mathrm{B} 2$ phases of $\mathrm{MgO}$ from $\mathrm{QM}$ is in very good agreement with experiment. The QM (DFT-GGA) EOS for the B1 and B2 phases of $\mathrm{MgO}$ were fitted to the MS-Q type force fields (qMS-Q) used successfully in describing phase transitions in silica. ${ }^{13}$ The qMS-Q FF is then used to calculate the EOS of $\mathrm{MgO}$ at finite temperature using NPT (constant $P$ and $T$ ) MD for $P$ up to 500 $\mathrm{GPa}$ and $T$ to $8000 \mathrm{~K}$.

Section II presents the QM (DFT-GGA) results for the B1 and $\mathrm{B} 2$ phases of $\mathrm{MgO}$ at $T=0 \mathrm{~K}$. Section III uses the QM results to obtain the parameters for the qMS-Q FF. Section IV uses the qMS-Q FF in NPT MD calculations of the $\mathrm{B} 1-\mathrm{B} 2$ and B1-liquid phase transition in $\mathrm{MgO}$. Finally, Sec. $\mathrm{V}$ draws some conclusions.

\section{II. $T=0$ EQUATION-OF-STATE: DFT CALCULATIONS}

\section{A. DFT-GGA calculations}

In this section we present first-principles calculations of the EOS for the $\mathrm{B} 1$ and $\mathrm{B} 2$ phases of $\mathrm{MgO}$. This corresponds to $T=0 \mathrm{~K}$ except that zero-point energy (ZPE) effects are not included. Our calculations are based on DFT, ${ }^{14-16}$ using GGA for the exchange-correlation energy functional. ${ }^{17}$ In these calculations, pseudopotentials are used to replace the core electrons $(1 s, 2 s, 2 p$ for $\mathrm{Mg}, 1 s$ for $\mathrm{O}) .{ }^{16}$ The total energies for various volumes are included in the supplementary material. [For convenience in refitting the FF, we have detailed the various numbers that might be useful and have made them available as supplementary material (see Ref. 18).]

Figure 1 shows the calculated pressure-volume curves for the B1 (circles) and B2 (squares) phases. Figure 2(a) shows the energy-volume curves for both phases, while Fig. 2(b) shows the enthalpy $H=U+P V$ calculated as a function of the pressure for both phases. Throughout this paper the vol- 


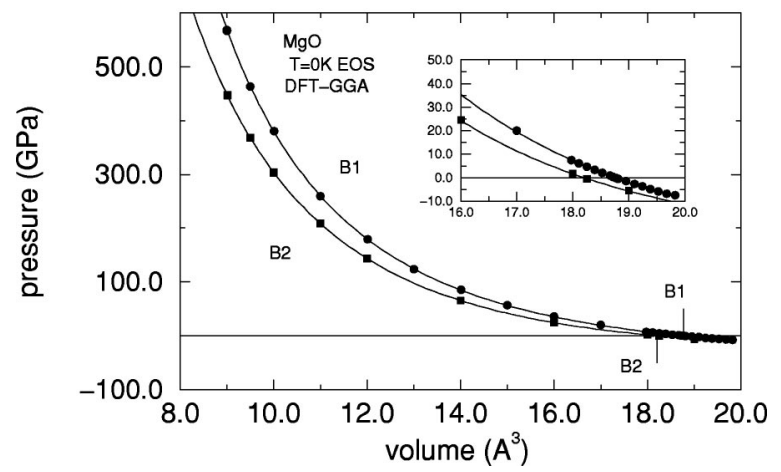

FIG. 1. Pressure as a function of volume for B1 (circles) and B2 (squares) phases of $\mathrm{MgO}$ from QM (using DFT-GGA). This corresponds to $T=0 \mathrm{~K}$ except that ZPE effects are excluded. The lines are the fit to a Birch-Murnaghan EOS [see Eq. (1)].

ume, energy, and enthalpy are quoted per formula unit, $\mathrm{MgO}$. Figure 2(b) shows a pressure-induced phases transition from B1 to B2 structure at a pressure of $P=400 \mathrm{GPa}$ (for $T=0$ ). This phase transition has not been observed experimentally up to $227 \mathrm{GPa}$, but no experimental results are available for higher pressures. Various theoretical predictions of this phase transition lead to transition pressures ranging from $1050 \mathrm{GPa}$ (Ref. 2) to $205 \mathrm{GPa}^{3}$ The most reliable of these calculations, ${ }^{6}$ which uses DFT with the local density approximation (LDA) rather than GGA, obtains $P_{B 1 B 2}$ $=450 \mathrm{GPa}$. The experimental EOS (at $295 \mathrm{~K}$ ) for the B1 phase compares well with the calculated EOS (at $0 \mathrm{~K}$ ).

\section{B. Birch-Murnaghan EOS}

We fitted the calculated pressure-volume data to a fifthorder Birch-Murnaghan (BM) EOS

$$
P=3 K_{0} f(1+2 f)^{5 / 2}\left(1+a_{1} f+a_{2} f^{2}+a_{3} f^{3}\right),
$$

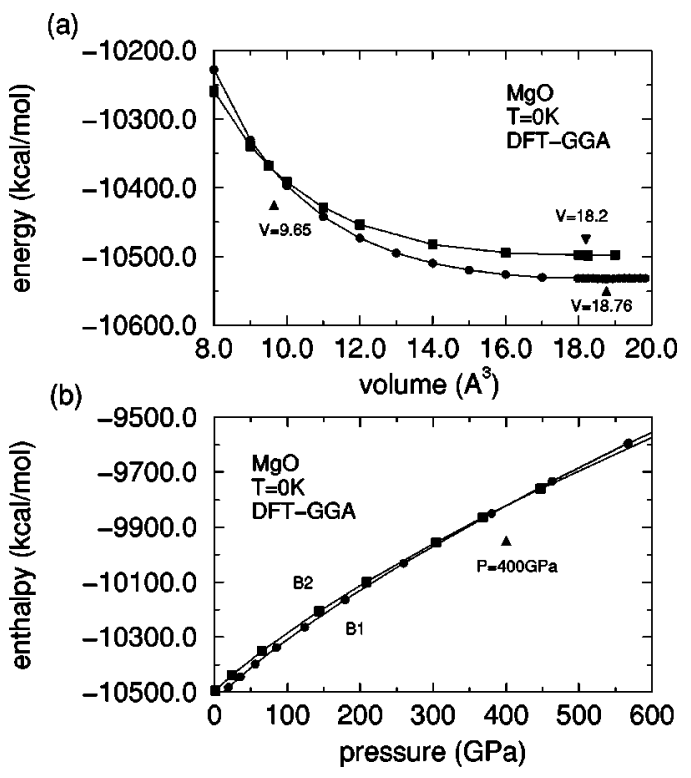

FIG. 2. (a) Energy as a function of volume for DFT-GGA calculations in Fig. 1. B1 (circles) and B2 (squares) phases of $\mathrm{MgO}$ at $T=0 \mathrm{~K}$. (b) Enthalpy as a function of pressure for the DFT-GGA calculations in (a) $(T=0 \mathrm{~K})$. where

$$
\begin{gathered}
f=\frac{1}{2}\left[\left(\frac{V_{0}}{V}\right)^{2 / 3}-1\right], \\
a_{1}=\frac{3}{2}\left(K_{0}^{\prime}-4\right), \\
a_{2}=\frac{3}{2}\left(K_{0} K_{0}^{\prime \prime}+K_{0}^{\prime}\left(K_{0}^{\prime}-7\right)+\frac{143}{9}\right), \\
a_{3}=\frac{1}{8}\left[9 K_{0}^{2} K_{0}^{\prime \prime \prime}+12\left(3 K_{0}^{\prime}-8\right) K_{0} K_{0}^{\prime \prime}\right. \\
\left.+\left(\left(3 K_{0}^{\prime}-16\right)^{2}+118\right) K_{0}^{\prime}-\frac{1888}{3}\right] .
\end{gathered}
$$

Here $V_{0}$ is the zero-pressure volume, $K_{T}=$ $-1 / V(\partial P / \partial V)_{T}$ is the isothermal bulk modulus (calculated at $V_{0}$ ), while $K_{T}^{\prime}$ and $K_{T}^{\prime \prime}$ denote the first and second derivatives of the bulk modulus with respect to pressure (evaluated at $V_{0}$ ).

The four parameters in this $\operatorname{EOS}\left(V_{0}, K_{0}, K_{0}^{\prime}\right.$, and $\left.K_{0}^{\prime \prime}\right)$ are displayed in Table I, together with other theoretical and experimental values. The line passing through the data points in Fig. 1 shows the fitted BM EOS. To estimate the errors in this BM EOS, we also fitted the BM EOS to these following volume ranges: 9-20 $\AA^{3}, 10-20 \AA^{3}$, and $11-20 \AA^{3}$. The differences in the fitted coefficients from these different ranges is used to estimate the uncertainty.

The experimental data shown in Table I by Jackson et al. ${ }^{19}$ was obtained from ultrasonic sound velocity measurements for pressures up to $3 \mathrm{GPa}$ which yields highprecision values for the bulk modulus and its pressure derivative, which were converted from adiabatic to isothermal conditions. We also show more recent results by Duffy et al. ${ }^{1}$ obtained from x-ray diffraction in diamond-anvil cell. The authors found that the high value of the bulk modulus in Ref. 1 is due to the static shear strength, and if this strength is taken into account a single equation of state can describe ultrasonic, shock and static compression data. ${ }^{1}$ The experimental value for $V_{0}$ is taken from Mao and Bell. ${ }^{20}$

Table I shows that the DFT-GGA EOS for B1 $\mathrm{MgO}$ is in good agreement with experiment. Previous theoretical predictions of the EOS have been reported by Karki et al. ${ }^{6}$ using DFT-LDA with pseudopotentials and by Mehl et al. ${ }^{4}$ using the all-electron linearized augmented plane-wave (LAPW) method, these results are also shown in Table I.

We also fitted the B2 pressure-volume data with a BM EOS. The results are displayed in Table II. Since the $\mathrm{MgO}$ B2 phase is not stable at low pressures, there are no experiments with which to compare.

\section{B1-B2 transformation pathway}

Since we are interested in studying the B1-B2 phase transition, we considered the Buerger lattice transformation at the pressure $P=420 \mathrm{GPa}$ and $T=0 \mathrm{~K}$ (note that the transition pressure from $\mathrm{B} 1$ to $\mathrm{B} 2$ is $400 \mathrm{GPa})$. Starting with the cubic unit cell for the $\mathrm{B} 1(\mathrm{NaCl})$ structure (with cell parameters $a=b=c$ and $\alpha=\beta=\gamma=90^{\circ}$ ), we changed the angles 
TABLE I. EOS parameters for the $\mathrm{B} 1(\mathrm{NaCl})$ phase of $\mathrm{MgO}$.

\begin{tabular}{|c|c|c|c|c|c|}
\hline & $V_{0} \quad\left(\AA^{3}\right)$ & $K_{T}(\mathrm{GPa})$ & $K_{T}^{\prime}$ & $K_{T}^{\prime \prime}$ & $K_{T}^{\prime \prime \prime}$ \\
\hline & \multicolumn{5}{|c|}{ Theory (minimization) } \\
\hline This work (DFT-GGA) & 18.76 & $161.5 \pm 1.5$ & $4.0 \pm 0.2$ & $-0.026 \pm 0.01$ & $0.0013 \pm 0.0006$ \\
\hline This work (qMS-Q FF) & 18.60 & $160.1 \pm 1.5$ & $4.5 \pm 0.04$ & $-0.035 \pm 0.01$ & $0.0027 \pm 0.0004$ \\
\hline DFT-LDA $^{a}$ & 19.20 & 159.7 & 4.25 & -0.026 & \\
\hline \multirow[t]{2}{*}{ LAPW $^{b}$} & 18.08 & 172 & 4.09 & & \\
\hline & \multicolumn{5}{|c|}{ Theory $(0 \mathrm{~K})$} \\
\hline This work (qMS-Q FF) & 18.80 & $151.5 \pm 1$ & $4.6 \pm 0.5$ & $-0.03 \pm 0.007$ & $0.0025 \pm 0.0003$ \\
\hline \multirow[t]{2}{*}{$\mathrm{PIB}^{\mathrm{c}}$} & 18.59 & 184.6 & 4.09 & -0.02 & \\
\hline & \multicolumn{5}{|c|}{ Theory (295 K) } \\
\hline This work (qMS-Q FF) & 18.87 & $145.0 \pm 0.2$ & $4.9 \pm 0.1$ & $-0.06 \pm 0.01$ & $0.004 \pm 0.0015$ \\
\hline PIB $(300 \mathrm{~K})^{\mathrm{c}}$ & 18.66 & 180.1 & 4.15 & -0.026 & \\
\hline \multirow[t]{2}{*}{$\operatorname{VIB}(300 \mathrm{~K})^{\mathrm{d}}$} & 18.70 & 153.11 & 4.68 & -0.05 & \\
\hline & \multicolumn{5}{|c|}{ Experiment $(295 \mathrm{~K})$} \\
\hline Ultrasound $(295 \mathrm{~K})^{\mathrm{e}}$ & 18.68 & $160 \pm 0.2$ & $4.15 \pm 0.1$ & & \\
\hline Diamond anvil $^{\mathrm{f}}$ & & $177 \pm 4$ & $4.0 \pm 0.1$ & & \\
\hline
\end{tabular}

${ }^{\mathrm{a}}$ Reference 6 .

${ }^{\mathrm{b}}$ Reference 4.

${ }^{\mathrm{c}}$ Reference 5 .

${ }^{\mathrm{d}}$ Reference 25 .

${ }^{\mathrm{e}}$ Reference 19 ( $V_{0}$ from Ref. 20).

${ }^{\mathrm{f}}$ Reference 1 .

in small steps to $\alpha=\beta=\gamma=109.47^{\circ}$, which leads to the $\mathrm{B} 2$ $(\mathrm{CsCl})$ structure. This transformation leads to an upper bound on the enthalpic barrier for the B1 to B2 phase transformation. Writing $\alpha=\beta=\gamma=90^{\circ}+19.47^{\circ} \lambda$, where $\lambda=0$ corresponds to the $\mathrm{B} 1$ structure and $\lambda=1$ corresponds to the $\mathrm{B} 2$ structure, we calculated the cell parameter $a=b=c$ leading to $P=420 \mathrm{GPa}$ for different values of $\lambda$. In this way we obtain the properties of the system for the Buerger transformation at $T=0 \mathrm{~K}$ and $P=420 \mathrm{GPa}$.

Figure 3 shows the energy [Fig. 3(a)] and enthalpy [Fig. 3(b)] from the QM as a function of the path variable $\lambda$ for $T=0 \mathrm{~K}$ and $P=420 \mathrm{GPa}$. We find that the QM enthalpy barrier is $\sim 6.8 \mathrm{kcal} / \mathrm{mol}$. Of course this is an upper bound on the actual transformation barrier which may involve more complicated intermediate structures and probably involves nucleation at surfaces or defects. The calculated shear stresses along the path are shown in Fig. 3(c). These shear stresses are quite large $(\sim 10 \mathrm{GPa})$, suggesting that deviatoric shear stresses of $\sim 10 \mathrm{GPa}$ might facilitate the phase transformation (which occurs at a large hydrostatic pressure of $\sim 400 \mathrm{GPa}$ ).

\section{III. qMS-Q FORCE FIELD}

In order to predict the properties of $\mathrm{MgO}$ at finite temperature and for imperfect crystals (including surfaces, defects, mixtures with other oxides, etc.) and liquids, we want to develop a FF that reproduces the QM EOS for the B1 and $\mathrm{B} 2$ phases of $\mathrm{MgO}$. We will use the MS-Q type FF which has been previously used to simulate phase transitions in silica. $^{13,21,22}$ The MS-Q FF allows the charges on individual atoms to change in response to the instantaneous environment of each particular atom. These charges are calculated using the charge equilibration (QEq) method of Rappé and Goddard. ${ }^{23}$ In addition to electrostatic interactions the MS-Q FF uses a Morse-Stretch two-body term

$$
U\left(r_{i j}\right)^{\mathrm{MS}}=D_{0}\left\{\exp \left[\gamma\left(1-\frac{r_{i j}}{R_{0}}\right)\right]-2 \exp \left[\frac{\gamma}{2}\left(1-\frac{r_{i j}}{R_{0}}\right)\right]\right\}
$$

to describe short-range Pauli repulsion forces and long-range attractions not included in the electrostatics. The parameters

TABLE II. EOS parameters for the $\mathrm{B} 2(\mathrm{NaCl})$ phase of $\mathrm{MgO}$.

\begin{tabular}{lcccc}
\hline \hline & $V_{0}\left(\AA^{3}\right)$ & $K_{T}(\mathrm{GPa})$ & $K_{T}^{\prime}$ & $K_{T}^{\prime \prime}$ \\
\hline & & \multicolumn{2}{c}{ Theory (minimization) } & \\
This work (DFT-GGA) & 18.2 & $145.5 \pm 1$ & $4.05 \pm 0.1$ & $-0.0215 \pm 0.004$ \\
This work (qMS-Q FF) & 18.11 & $144.3 \pm 1$ & $4.6 \pm 0.05$ & $-0.04 \pm 0.005$ \\
& & & & \\
& & & Theory $(0 \mathrm{~K})$ & $-0.03 \pm 0.005$ \\
This work (qMS-Q FF) & 18.46 & $136.9 \pm 1$ & $4.5 \pm 0.1$ & \\
\hline \hline
\end{tabular}


of the Morse potential, $D, \gamma$, and $R_{0}$ depend on the nature of the atoms $i$ and $j$. Thus, we have three parameters for the $\mathrm{Mg}-\mathrm{Mg}$ interaction, three for $\mathrm{Mg}-\mathrm{O}$, and three for $\mathrm{O}-\mathrm{O}$. Thus, nine parameters were optimized to describe the energy and volume for both the B1 and B2 phases for pressure from 0 to $500 \mathrm{GPa}$ (we did not modify the QEq parameters). The final FF is referred to as qMS-Q, the q denoting the QM origin of the parameters.

The electrostatic interactions were calculated using the accuracy bonded convergence acceleration Ewald method ${ }^{24}$ with parameters chosen for maximal convergence. Thus, at 0 (300) GPa we used an Ewald exponent of $\eta=1.559$ (1.329) $\AA$, a real-space cutoff of 4.96 (4.27) $\AA$ and a reciprocal space cutoff of $1 / \lambda=0.794(0.947) \AA$. The Morse interaction in Eq.
(5) was cutoff using a cubic spline starting at $11 \AA$ and ending at $15 \AA$.

\section{A. Optimization of qMS-Q}

The MS-Q parameters for the $\mathrm{Mg}-\mathrm{Mg}, \mathrm{O}-\mathrm{O}$, and $\mathrm{Mg}-\mathrm{O}$ Morse potentials were chosen to reproduce the following $\mathrm{QM}$ results for $\mathrm{MgO}$ : (i) Energy-volume curves for $\mathrm{B} 1$ and B2 phases, (ii) pressure-volume curves for B1 and B2 phases, (iii) bulk modulus for both phases, and (iv) shear stresses along the Buerger path.

This was accomplished by using an optimization algorithm based on simulated annealing to find the set of parameters minimizing the cost function in Eq. (6):

$$
\begin{aligned}
C= & \sum_{V_{i}}^{9}\left\{\left[P_{Q M}^{B 1}\left(V_{i}\right)-P_{F F}^{B 1}\left(V_{i}\right)\right]^{2} W_{p}^{B 1}\left(V_{i}\right)+\left[E_{Q M}^{B 1}\left(V_{i}\right)-E_{F F}^{B 1}\left(V_{i}\right)\right]^{2} W_{e}^{B 1}\left(V_{i}\right)\right\}+\sum_{V_{i}}^{9}\left\{\left[P_{Q M}^{B 2}\left(V_{i}\right)-P_{F F}^{B 2}\left(V_{i}\right)\right]^{2} W_{p}^{B 2}\left(V_{i}\right)\right. \\
& \left.+\left[E_{Q M}^{B 2}\left(V_{i}\right)-E_{F F}^{B 2}\left(V_{i}\right)\right]^{2} W_{e}^{B 2}\left(V_{i}\right)\right\}+\sum_{\alpha}^{6} \sum_{i=z x}^{x y}\left[\sigma_{i}^{Q M}(\alpha)-\sigma_{i}^{F F}(\alpha)\right]^{2} W_{\sigma}(\alpha)+\left\{\left[P_{Q M}^{B 1}\left(V_{1}\right)-P_{Q M}^{B 1}\left(V_{2}\right)\right]\right. \\
& \left.-\left[P_{F F}^{B 1}\left(V_{1}\right)-P_{F F}^{B 1}\left(V_{2}\right)\right]\right\}^{2} W_{B}^{B 1}+\left\{\left[P_{Q M}^{B 2}\left(V_{1}\right)-P_{Q M}^{B 2}\left(V_{2}\right)\right]-\left[P_{F F}^{B 2}\left(V_{1}\right)-P_{F F}^{B 2}\left(V_{2}\right)\right]\right\}^{2} W_{B}^{B 2} \\
& +\sum_{V_{i}}^{2}\left\{\left[\Delta P_{Q M}\left(V_{i}\right)-\Delta P_{F F}\left(V_{i}\right)\right]^{2} W_{\Delta P}\left(V_{i}\right)+\left[\Delta E_{Q M}\left(V_{i}\right)-\Delta E_{F F}\left(V_{i}\right)\right]^{2} W_{\Delta E}\left(V_{i}\right)\right\},
\end{aligned}
$$

where $Q M$ denotes the DFT-GGA results (Figs. 1, 2, and 3) and $F F$ is the qMS-Q FF. The first sum in the cost function runs over nine different volumes for the B1 phase, the second sum runs over nine volumes for the B2 phase. The DFTGGA energies and pressures calculated for various volumes are included in the supplementary material, see Ref. 18. All energies are calculated relative to the energy of the $\mathrm{B} 1$ phase at $V=19.817 \AA^{3}$. The third sum in Eq. (6) runs over six points along the Buerger path, $\sigma_{i}$ denotes the shear stress and $i$ runs over $z y, z x$, and $x y$. The variable $\alpha$ denotes the cell angles and lengths along the path. The shear stresses along the Buerger path are also included in the supplementary material. The fourth and fifth terms in the cost function contain pressure differences at different volumes and are related to the bulk modulus of each phase. We took $V_{1}=19.817 \AA^{3}$ and $V_{2}=15 \AA^{3}$ for $\mathrm{B} 1$ and $V_{1}=19 \AA^{3}$ and $V_{2}=14 \AA^{3}$ for B2. In the last term $\Delta P\left(V_{i}\right)$ and $\Delta E\left(V_{i}\right)$ denote pressure and energy difference between B1 and B2 phases at volume $V_{i}$. In the last term the sum runs over $V=9 \AA^{3}$ and $V=11 \AA^{3}$.

The parameters $W$ entering the cost function make it a dimensionless quantity and act as weights for the different terms entering the optimization algorithm. The weights for the pressure-volume relations $W_{p}\left(V_{i}\right)$ are taken to be $W_{p}\left(V_{i}\right) \propto 1 /\left(\left|P_{Q M}\left(V_{i}\right)\right|+P_{\text {shift }}\right)$. In this way we give more importance to the low-pressure points, which are important in the value of the zero-pressure volume $\left(V_{0}\right)$ and bulk modulus. We took $P_{\text {shift }}=200 \mathrm{GPa}$ for B1 and $P_{\text {shift }}=900$ $\mathrm{GPa}$ for B2. The weights for the pressure-volume terms are normalized to $\Sigma_{V_{i}}^{9} W_{p}\left(V_{i}\right)=1$ for the B1 structure and to $\Sigma_{V_{i}}^{9} W_{p}\left(V_{i}\right)=2$ for the $\mathrm{B} 2$ structure. The energy-volume weights $W_{e}$, which do not depend on $V_{i}$, are normalized to $\Sigma_{V_{i}}^{9} W_{e}=10$ for $\mathrm{B} 1$ and $\Sigma_{V_{i}}^{9} W_{e}=0.2$ for B2. The $W_{\sigma}$ are constant and satisfy: $\Sigma_{\alpha}^{6} W_{\sigma}=0.6$. We took $W_{B}^{B 1}=100$ and $W_{B}^{B 2}=0.01$. Finally, we took $W_{\Delta P}\left(11 \AA^{3}\right)=0.01$, $W_{\Delta P}\left(9 \AA^{3}\right)=0.1, W_{\Delta E}\left(11 \AA^{3}\right)=5$, and $W_{\Delta E}\left(9 \AA^{3}\right)=5$.

While the specific values of weights are not very important we chose them taking into account the following guidelines. The force field should give good results for both phases, paying particular attention to the pressure at which the phase transition from B1 to B2 occurs. Furthermore, we found that a good agreement in the B1 bulk modulus with DFT-GGA results gives accurate melting temperature, consequently the weight related to this quantity is high. The optimized qMS-Q FF parameters are listed in Table III.

Figure 4 compares the EOS at $T=0 \mathrm{~K}$ for the qMS-Q FF (filled symbols) with the DFT results (open symbols). We see that the agreement between the qMS-Q FF and DFTGGA is very good for both the B1 and B2 phases. For very high compression $V \sim V_{0} / 2$ our FF slightly overestimates the pressure of the $\mathrm{B} 2$ phase.

Figure 5 shows the energy-volume and enthalpy-pressure curves for the qMS-Q FF. Figure 5(b) shows that the $T=0 \mathrm{~K}$ phase transition is at $\sim 400 \mathrm{GPa}$ for both FF and QM. This is consistent with experiments which show no evidence of a transformation at $P$ up to $227 \mathrm{GPa}$.

Figure 6 shows the energy, enthalpy, and shear stresses along the Buerger path as a function of the path variable $\lambda$ (defined above). These quantities are calculated at $T=0 \mathrm{~K}$ 

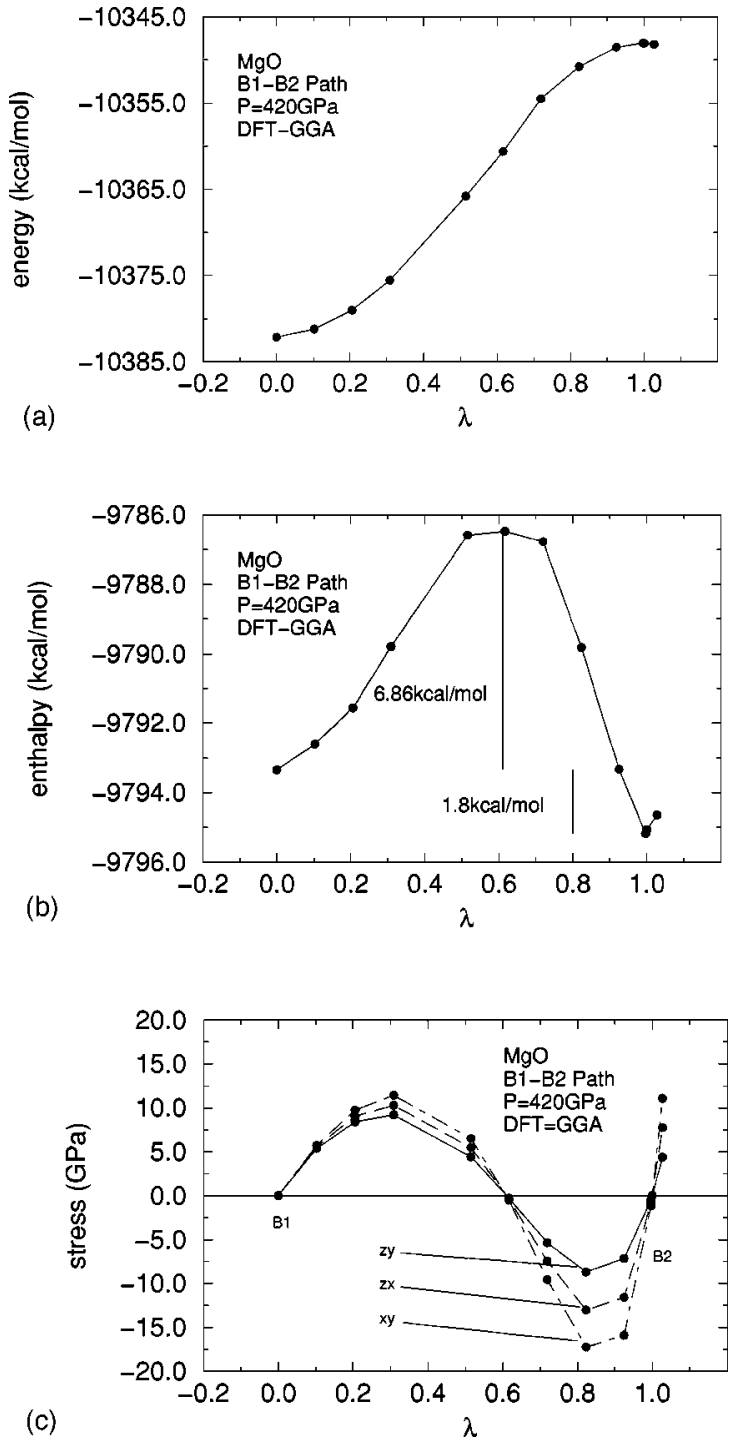

FIG. 3. B1-B2 Buerger path, at $T=0 \mathrm{~K}$ and $P=420 \mathrm{GPa}$ using DFT-GGA. (a) energy as a function of the path variable $\lambda$, (b) enthalpy as a function of $\lambda$, and (c) shear stresses as a function of the path variable $\lambda$. Solid lines denote the $z y$ component, dotted lines the $x z$ and dashed lines denote the $x y$ component.

and $P=420 \mathrm{GPa}$, the same temperature and pressure used for the DFT-GGA calculations shown in Fig. 3. The general behavior obtained for the FF is similar to the one obtained using QM. Quantitatively the energy barrier for the FF is about $60 \%$ of the QM value $(4.25 \mathrm{kcal} / \mathrm{mol} \mathrm{vs} 6.8 \mathrm{kcal} / \mathrm{mol})$ and the shear stresses are about $60 \%$ of the QM results.

\section{B. $\mathrm{Mg}$ and $\mathrm{O}$ charges from charge equilibration}

A key feature of the qMS-Q FF is that the charges of the individual atoms depend on their instantaneous environment.

TABLE III. qMS-Q parameters for $\mathrm{MgO}$

\begin{tabular}{lccc}
\hline \hline & $D(\mathrm{kcal} / \mathrm{mol})$ & $\gamma$ & $R_{0}(\AA)$ \\
\hline $\mathrm{Mg}-\mathrm{O}$ & 14.00 & 7.7699 & 1.9851 \\
$\mathrm{Mg}-\mathrm{Mg}$ & 2.99 & 8.0026 & 3.8723 \\
$\mathrm{O}-\mathrm{O}$ & 0.52 & 8.0160 & 4.2585 \\
\hline \hline
\end{tabular}

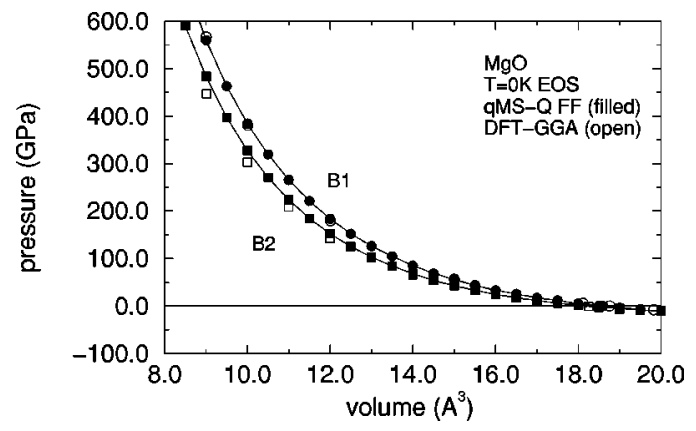

FIG. 4. Pressure-volume curves for $\mathrm{MgO}$ at $T=0 \mathrm{~K}$ from qMS-Q FF (filled symbols) and DFT-GGA (empty symbols). Circles denote B1 phase and squares denote B2.

This leads to slightly different charges for the B1 and B2 phases, which change with pressures as shown in Table IV. The charges are calculated using the charge equilibration method, ${ }^{23}$ Table $\mathrm{V}$ shows the parameters used in the charge calculation.

\section{Zero-point energy corrections}

To compare our results to experiment even at $T=0 \mathrm{~K}$, we must take into account ZPE for the crystal. To do this we need to calculate the vibrational modes through the Brillouin zone (BZ).

The ZPE is given by

$$
U_{\mathrm{tot}}^{\mathrm{ZPE}}=\sum_{s} \int_{f b z} \frac{1}{2} \hbar \omega_{s}(\mathbf{k}) d^{3} k
$$

where the sum over all six phonon modes $\omega_{s}(\mathbf{k})$ for each $\mathbf{k}$ in the BZ. (We approximate the integral over the BZ using $5 \times 5 \times 5$ points in the $\mathrm{BZ}$ for the cubic cell.) This calculation is carried out for each volume. The ZPE (included in the
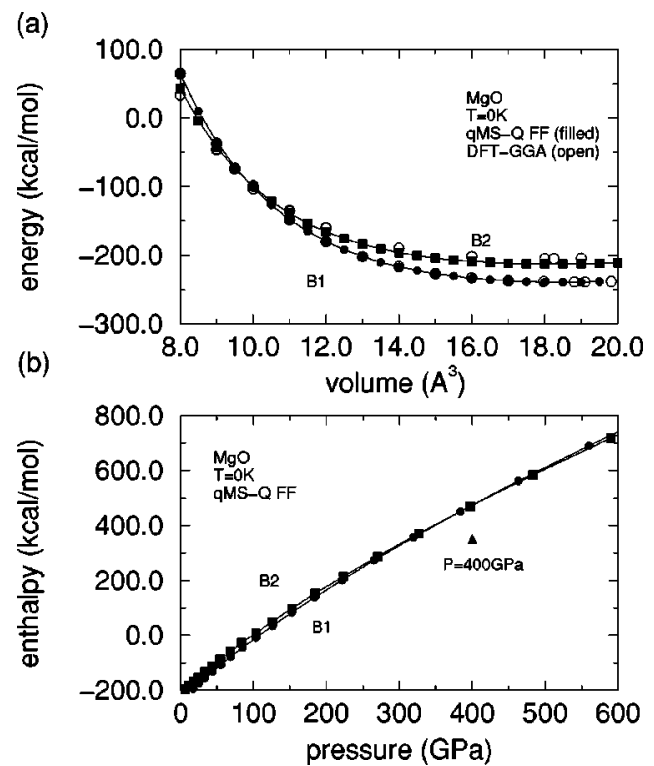

FIG. 5. (a) Energy versus volumes at $T=0 \mathrm{~K}$ for the $\mathrm{B} 1$ (circles) and B2 (squares) phase using the qMS-Q FF. (b) Enthalpy versus pressure at $T=0 \mathrm{~K}$ for $\mathrm{B} 1$ (circles) and B2 (squares). 

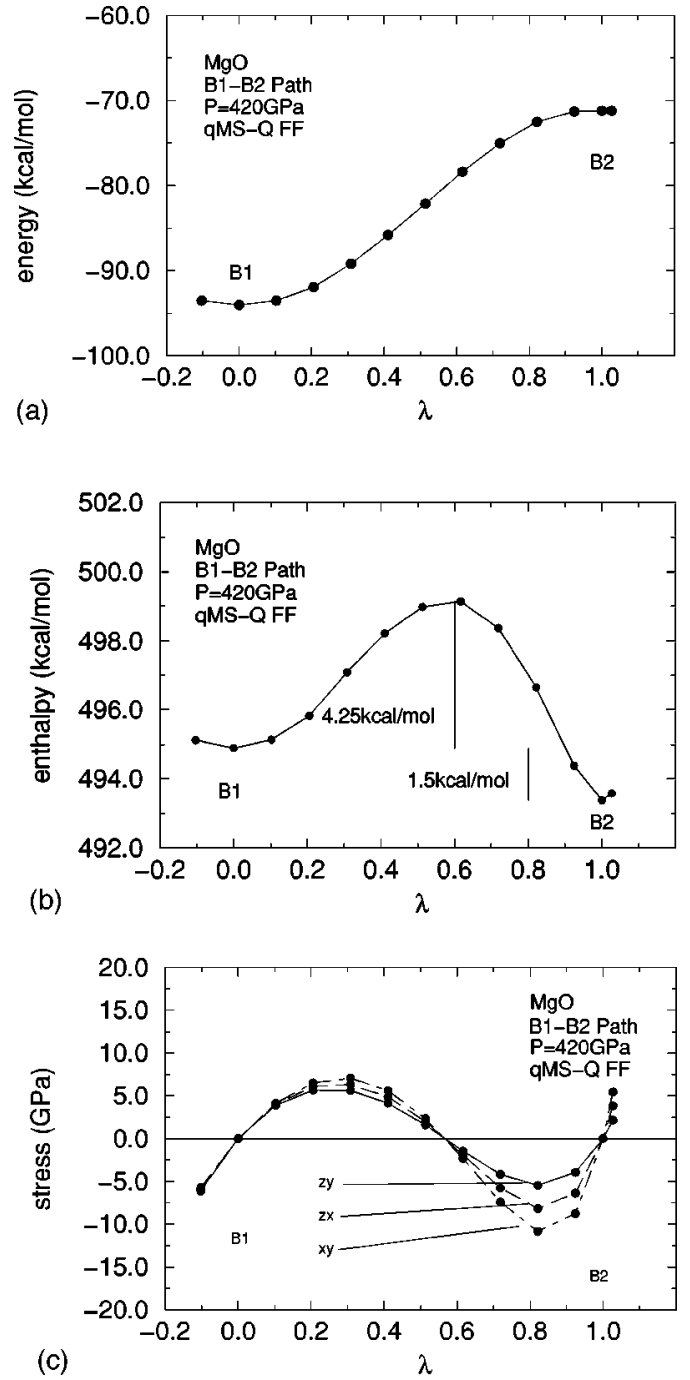

FIG. 6. B1-B2 Buerger path (at $T=0 \mathrm{~K}$ and $P=420 \mathrm{GPa}$ ) using qMS-Q FF. (a) energy as a function of the path variable $\lambda$, (b) enthalpy as a function of $\lambda$, and (c) shear stresses as a function of the path variable $\lambda$. Solid lines denote the $z y$ component, dotted lines the $x z$ and dashed lines denote the $x y$ component.

supplementary material) must be added to the QM energy or the FF energy to obtain the total energy of the system at $T$ $=0 \mathrm{~K}$,

$$
U_{\text {tot }}(0 \quad \mathrm{~K})=U_{q M S-Q F F}+U_{\text {tot }}^{\mathrm{ZPE}} .
$$

Including ZPE in the qMS-Q FF calculations increases the

TABLE IV. Mg charges for different pressures for B1 and B2 phases of $\mathrm{MgO}$.

\begin{tabular}{ccc}
\hline \hline$P(\mathrm{GPa})$ & $\mathrm{B} 1$ & $\mathrm{~B} 2$ \\
\hline 0 & 0.7078 & 0.7017 \\
50 & 0.7141 & 0.7092 \\
100 & 0.7162 & 0.7123 \\
200 & 0.7180 & 0.7157 \\
300 & 0.7193 & 0.7169 \\
400 & 0.7198 & 0.7182 \\
\hline \hline
\end{tabular}

TABLE V. QEq parameters used to obtain charges.

\begin{tabular}{lccc}
\hline \hline & $\chi(\mathrm{eV})$ & $\mathrm{J}(\mathrm{eV})$ & $R(\AA)$ \\
\hline $\mathrm{O}$ & 8.741 & 6.682 & 0.669 \\
$\mathrm{Mg}$ & 3.951 & 3.693 & 1.5 \\
\hline \hline
\end{tabular}

zero-pressure volume by $\sim 1 \%$ to $V=18.8 \AA^{3}$ and reduces the bulk modulus to $B=152.7 \mathrm{GPa}$, these results are also included in Table I.

\section{The EOS at $295 \mathrm{~K}$}

Using the qMS-Q FF in NPT MD simulations, we calculated the EOS at $T=295 \mathrm{~K}$. The zero-pressure volume, bulk modulus, and its derivatives (see Table I) are in good agreement with the experimental values. The EOS predicted for $T=295 \mathrm{~K}$ is shown in Fig. 7 together with the experimental curve. ${ }^{19}$ This experimental EOS was obtained by using the bulk modulus and its first and second derivatives obtained by Jackson et al. ${ }^{19}$ from ultrasonic sound velocity measurements at $T=295 \mathrm{~K}$ for pressures up to $3 \mathrm{GPa}$. It can be seen the our results compare well with the experiment. Previous $T=300 \mathrm{~K}$ results shown in Table I were obtained using the potential-induced breathing model (PIB) with the quasiharmonic approximation, ${ }^{5}$ and the variational-induced breathing model (VIB) with MD simulations. ${ }^{25}$

\section{THE PHASE DIAGRAM FOR B1-B2 AND B1-LIQUID}

We used the qMS-Q FF to investigate the B1-B2 and B1-liquid phase coexistence curves for $\mathrm{MgO}$.

\section{A. B1-B2 phase simulations}

Consider first the B1-B2 phase boundary. In order to calculate such properties as volume, enthalpy, and energy, as a function of $T$ and $P$, we carried out NPT MD simulations using the Nosé thermostat and the Rahman-Parrinello barostat. ${ }^{26}$ These calculations used a cubic periodic simulation cell with $8 \times 3^{3}=216$ atoms for B1 and $2 \times 4^{3}=128$ atoms for B2. For each $T$ and $P$ point we performed a 15 ps MD simulation, but used only the last $10 \mathrm{ps}$ for predicting properties (the first $5 \mathrm{ps}$ is to equilibrate at the new $T$ and $P$ ). The time step used to integrate the equation of motion was $\tau=0.001 \mathrm{ps}$ for $T<3000 \mathrm{~K}$ and $\tau=0.0005 \mathrm{ps}$ for higher temperatures.

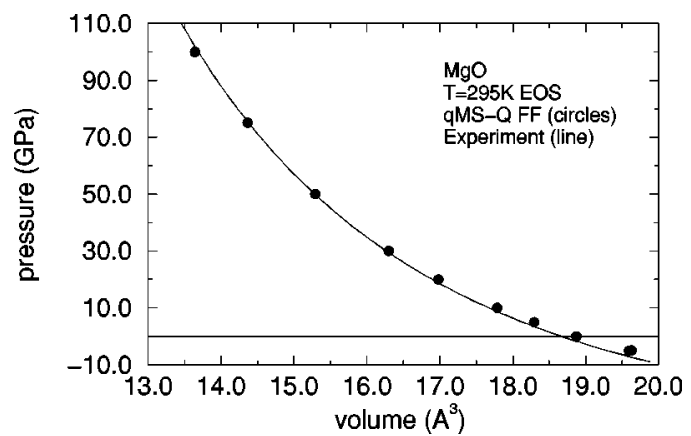

FIG. 7. EOS for B1 phase at $T=295 \mathrm{~K}$ using qMS-Q FF. The line shows experimental result of Jackson et al. (Ref. 19). 
(a)

(b)
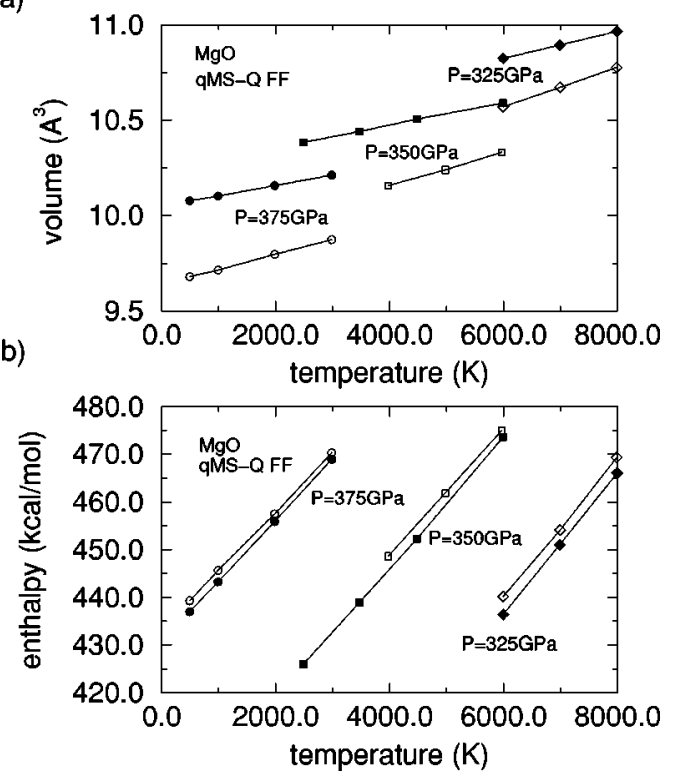

FIG. 8. (a) Volume and (b) enthalpy of B1 and B2 phases as a function of temperature for different pressures. Full symbols denote B1 structure while empty symbols denote B2 phase. This is based on the qMS-Q FF.

In this way, we obtained the difference in enthalpy and volume between the two solid phases as a function of $T$ for different pressures $P=375,350$, and $325 \mathrm{GPa}$. Figure 8 shows the volume [Fig. 8(a)] and enthalpy [Fig. 8(b)] for both phases as a function of temperature for pressures in the range of 400 to $300 \mathrm{GPa}$. As explained below, this data together with the Clausius-Clapeyron equation can be used to obtain the phase boundary between B1 and B2.

\section{B. B1-melt phase simulations}

To study the melting of $\mathrm{MgO}$ as a function of pressure, we started with the crystal of B1 at low temperatures and carried out NPT MD at a series of temperatures, increasing the temperature by $250 \mathrm{~K}$ every $15 \mathrm{ps}$. Of the $15 \mathrm{ps}$ at each $T$ and $P$ point, the first 5 ps was considered to be equilibration so that the calculated $V$ and $H$ were averaged only over last $10 \mathrm{ps}$. We continued this heating process until $\sim 400 \mathrm{~K}$ beyond the melting point. We then cooled the liquid to the range of temperatures at which we wanted to calculate the changes in enthalpy and volume between the melt and the solid. Figure 9 shows a typical volume-temperature curve using this procedure during the heating and cooling processes and it was obtained for $P=0 \mathrm{GPa}$. The experimental value of the zero-pressure melting temperature obtained by Zerr and Boehler is $3040 \pm 100 \mathrm{~K}$. This value is close to the melting temperature $(3100 \pm 100 \mathrm{~K})$ obtained from our simulations. Previous simulations lead to zero pressure melting temperatures of $3300 \mathrm{~K},{ }^{7} 3200,{ }^{8}$ and the range $3100-3250$ $\mathrm{K},{ }^{9}$ in agreement with our results and experiment.

With this procedure we obtained (Fig. 10) the volume and enthalpy for the liquid (empty symbols) and the solid (full symbols) as a function of temperature for various pressures $(P=0,10,25,50,100,200) \mathrm{GPa}$. For clarity not all pressures calculated are shown in Fig. 10.

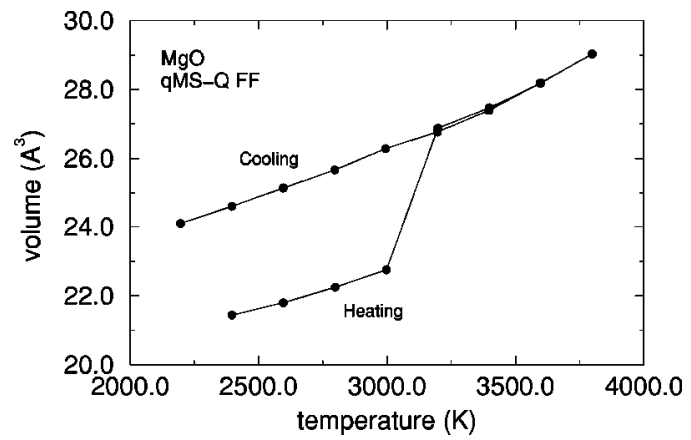

FIG. 9. Volume as a function of temperature for the B1 phase (lower branch) and liquid (upper branch). For $P=0 \mathrm{GPa}$ using qMS-Q FF.

\section{Determination of phase boundaries}

The calculation of accurate phase boundaries from MD is difficult. Typically MD leads to superheating of the solid and undercooling of the liquid due to the finite size of the periodic cell and the absence of defects and nucleation centers. The rapid heating and quenching rates causes hysteresis, making it difficult to specify the precise temperature corresponding to the phase transition. One approach to avoid this problem is to calculate the free energy by thermodynamic integration, ${ }^{27}$ or by two-phase simulation techniques. ${ }^{9}$ Instead, we choose to find the phase coexistence curve by using the Clausius-Clapeyron equation,

$$
\frac{d P}{d T}=\frac{1}{T} \frac{\Delta H}{\Delta V}
$$

which relates the derivative of the coexistence curve between two phases to the change in enthalpy and volume between the phases.
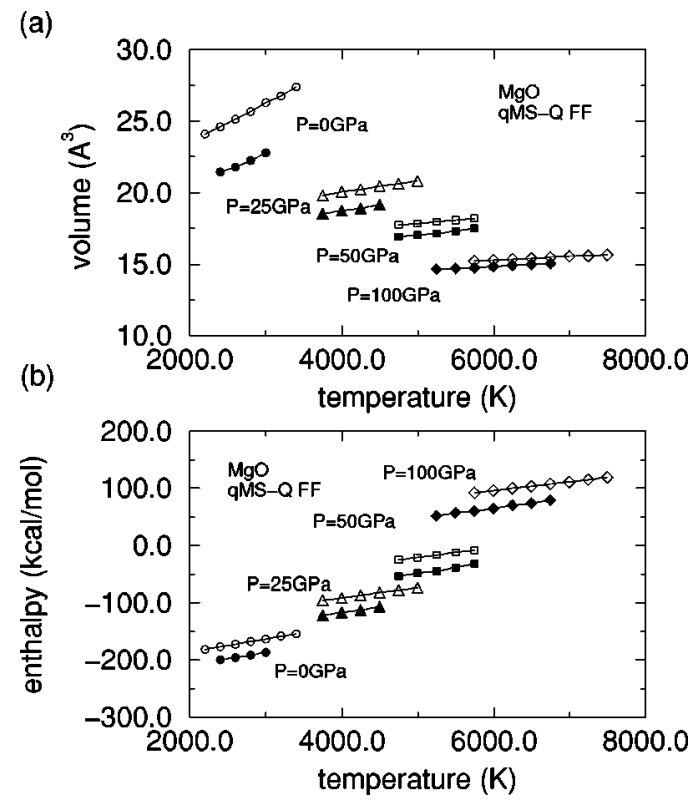

FIG. 10. (a) Volume and (b) enthalpy for the B1 and liquid phases as a function of temperature for different pressures. Full symbols denote B1 structure while empty symbols denote the liquid. 


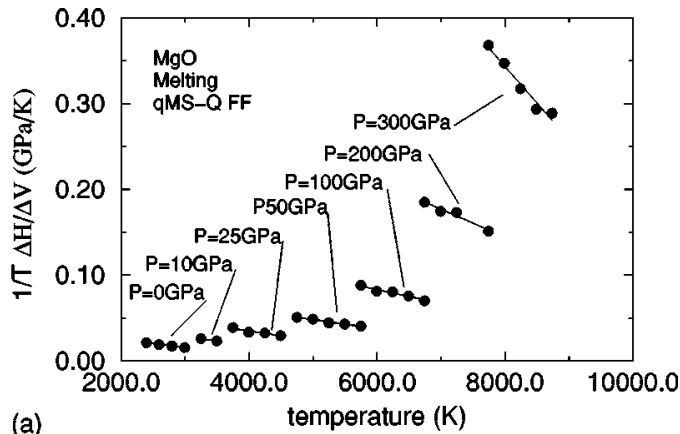

(a)
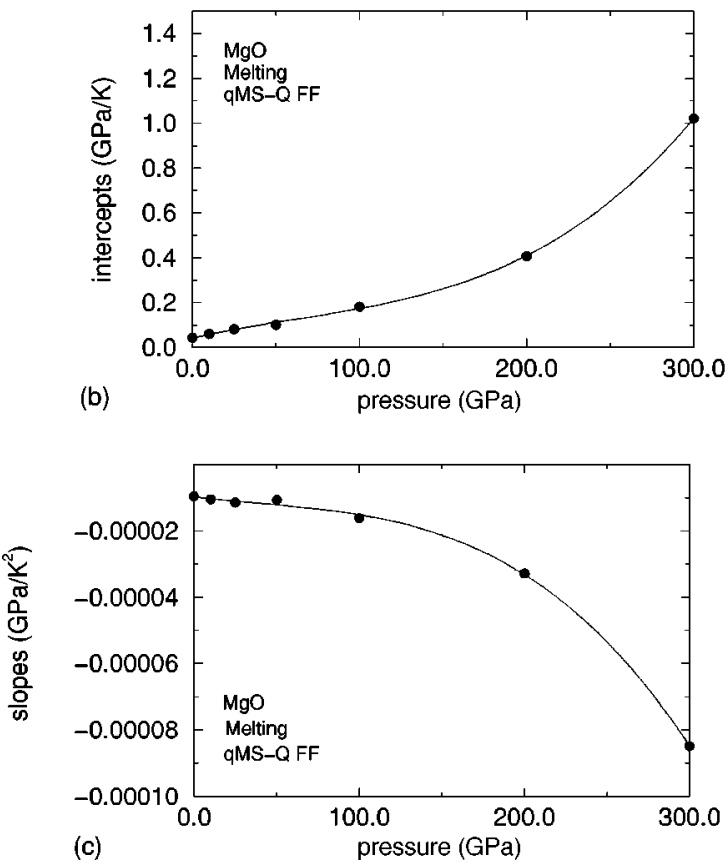

FIG. 11 . MgO melting. (a) $1 / T \Delta H / \Delta V$ as a function of temperature for different pressures, obtained from MD simulations using qMS-Q FF, the lines denote linear fits to the data (circles). (b) Intercepts $(T=0 \mathrm{~K})$ of the linear fits to the $1 / T \Delta H / \Delta V$ data as a function of pressure (circles), the line shows a third-order polynomial fit. (c) Slopes of the linear fits to the $1 / T \Delta H / \Delta V$ data as a function of pressure (circles), the line shows a third-order polynomial fit.

We devised the following procedure to calculate the B1-melt coexistence curve. Our MD calculations lead to $\Delta H$ and $\Delta V$ for the melting phase transitions as a function of temperature for various pressures. Figure 11(a) shows $[d P / d T]_{B 1-\text { melt }}=1 / T \Delta H / \Delta V$ for the B1-liquid phase transition as a function of temperature for different pressures, together with linear fits to the data. Thus, we know the derivative of the coexistence curve for the set of pressures shown, for different temperatures ranges. In order to accurately integrate Clausius-Clapeyron equation we would like to know $[d P / d T]_{B 1-\text { melt }}$ for all pressures. Figures $11(\mathrm{~b})$ and $11(\mathrm{c})$ show the intercepts (slopes) of the linear fits to our $[d P / d T]_{B 1-\text { melt }}$ data as a function of pressure. Both the intercepts and the slopes can be smoothly fitted with cubic polynomials [lines in Figs. 11(b) and 11(c)], which we will denote $\phi_{1}(P)$ and $\phi_{2}(P)$, respectively. In this way the slope of the coexistence curve, for any pressure, is given by

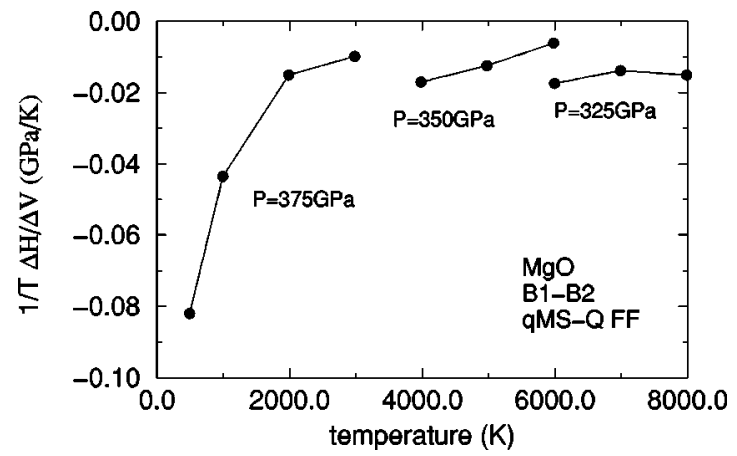

FIG. 12. B1-B2 phase transition. $1 / T \Delta H / \Delta V$ as a function of temperature for different pressures, obtained from MD simulations using qMS-Q FF.

$$
\left[\frac{d P}{d T}\right]_{B 1-\text { melt }}=\phi_{1}(P)+\phi_{2}(P) T,
$$

where $\quad \phi_{1}(P)=4.1303798810^{-2}+1.7063269210^{-3} P$ $-8.4135821510^{-6} P^{2}+4.5393834610^{-8} P^{3}$ and $\phi_{2}(P)=$ $-9.6718954410^{-6}-6.3051275410^{-8} P$ $+4.3311524710^{-10} P^{2}-3.5245486310^{-12} P^{3}$.

Equation (11) can be integrated exactly to obtain the coexistence curve, which leads to a family of curves for different initial conditions.

Consider now the B1-B2 phase transition. In Fig. 12 we show $[d P / d T]_{B 1-B 2}=1 / T \Delta H / \Delta V$ as a function of temperature for different pressures, namely $P=375,350$, and 325 $\mathrm{GPa}$. In this case we use a different procedure to estimate the coexistence curve. Consider a set of trial temperatures $T_{i}$ for the coexistence curve at pressure $P_{i}$ (ordered such that $P_{i}$ $\left.>P_{i+1}\right)$. At each $T_{i}$ we use Eq. (9) to predict the expected coexistence temperature $T_{i-1}^{\prime}$ at $P_{i-1}$ and $T_{i+1}^{\prime}$ at $P_{i+1}$. To make the best estimate for the coexistence curve, we find the set $\left\{T_{i}\right\}$ minimizing the following cost function:

$$
C=\sum_{i=2}^{\prime}\left(T_{i-1}-T_{i-1}^{\prime}\right)^{2}+\left(T_{i+1}-T_{i+1}^{\prime}\right)^{2}
$$

where the prime in the sum indicates that the term with $T_{i+1}$ is not taken into account if $P_{i}=325 \mathrm{GPa}$. This procedure was used to estimate the B1-B2 coexistence curve. Note that in this case we know one point of the coexistence curve: at $T$ $=0 \mathrm{~K}$ the transition pressure is $P=400 \mathrm{GPa}$.

The phase coexistence curves calculated with the abovementioned procedures are shown in Fig. 13. The full line for the B1-liquid boundary was obtained starting the integration of the Clausius-Clapeyron equation (11) from $P=0 \mathrm{GPa}$ and $T=3100 \mathrm{~K}$, which is our estimate of the zero-pressure melting temperature. The dashed lines were obtained starting with $T=3050 \mathrm{~K}$ and $T=3150 \mathrm{~K}$, at zero pressure and represent an estimate of the error in the coexistence curve. This uncertainty in the melting curve depends on our estimate of the error of the melting temperature at zero pressure, which can be greater than $100 \mathrm{~K}$ due to the difficulties in determining phase transitions in MD simulations mentioned in the Introduction.

Note that the B1-B2 coexistence curve has very low curvature, which means that the approximation used in integrat- 


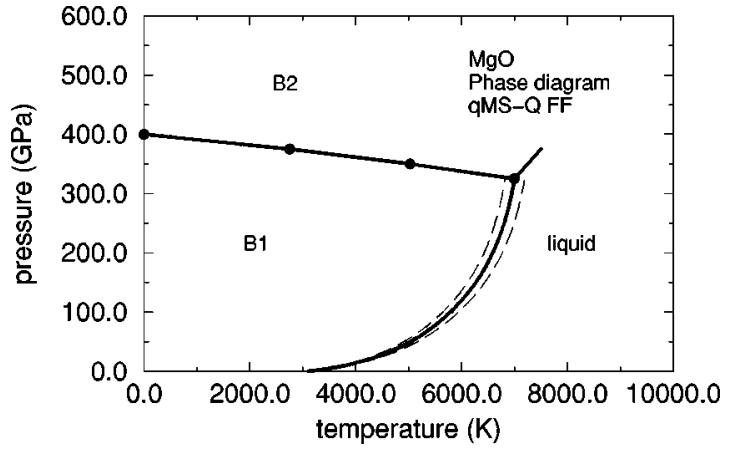

FIG. 13. Phase diagram of $\mathrm{MgO}$ according to $\mathrm{qMS}-\mathrm{Q}$ force field. The B1-B2, B1-liquid, and B2-liquid phase coexistence curves are shown.

ing the Clausius-Clapeyron equation, coming from the fact the we know the $d P / d T$ only for a set of pressures, is very good.

The B2-liquid coexistence curve was calculated from a MD melting simulation at $P=350 \mathrm{GPa}$ starting from a crystal in the B2 structure. We calculated $(d P / d T)_{B 2-\text { melt }}$ $=1 / T \Delta H / \Delta V$ as a function of temperature to determine the B2-melt phase boundary.

\section{Discussion of phase diagram}

The temperature dependence of the melting phase transition has been studied by several groups. ${ }^{7-9}$ This is, we believe, the first calculation of the B1-B2 phase coexistence for $\mathrm{MgO}$ at finite temperature.

In Fig. 14 we compare our results for the melting curve with the experimental values obtained by Zerr and Boehler. ${ }^{10}$ Our calculations lead to a melting temperature at $P=0 \mathrm{GPa}$, of $T_{\mathrm{MP}}(0 \mathrm{GPa}) \sim 3100 \pm 50 \mathrm{~K}$, which is in good agreement with the experimental value of Zerr and Boehler ${ }^{10}$ (3040 $\pm 100 \mathrm{~K})$. For higher pressures our melting temperature is higher than the experimental result. For example, at $P=25$ GPa we find $T_{\mathrm{MP}}=4400 \mathrm{~K} \pm 200 \mathrm{~K}$ slightly higher than the experimental result of $T_{\mathrm{MP}}(25 \mathrm{GPa}) \sim 3700 \pm 300 \mathrm{~K}$, see Fig. 14. These temperatures are lower than those obtained in other recent simulations, ${ }^{8,9}$ where $T_{\mathrm{MP}}(25 \mathrm{GPa}) \sim 5000 \mathrm{~K}$. From our calculations the slope of the B1-liquid coexistence curve, at zero pressure, is $\sim 0.0113 \mathrm{GPa} / \mathrm{K}$. This value is lower than the experimental slope $\sim 0.0278 \mathrm{GPa} / \mathrm{K}$ (Ref. 10) and higher than the one obtained in previous simulations $\sim 0.0088 \mathrm{GPa} / \mathrm{K}$ (Ref. 8).

Solid-solid phase transitions are difficult to study via computer simulations, because the small size of the simulation cell and the lack of defects for nucleating the new phase lead to long-lived metastable states. This problem is most noticeable at the ultrahigh pressures of interest here. Our MD simulations of the B1 and B2 phases never exhibited a transition between $\mathrm{B} 1$ and $\mathrm{B} 2$ for the pressures and temperatures studied. We obtained the B1 to B2 coexistence curve from thermodynamic quantities extracted from the simulation, but we could not study directly the kinetics for this phase transformation.

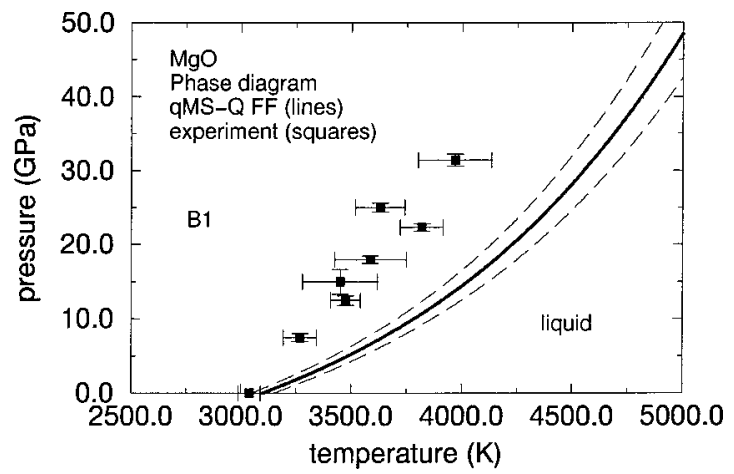

FIG. 14. Melting curve of MgO. Lines show qMS-Q FF results for the B1-liquid coexistence curve. Squares are experimental results from Ref. 10.

\section{SUMMARY}

We presented here a strategy for determining equationsof-state and phase diagrams from first-principles QM calculations at $T=0 \mathrm{~K}$.

Using the QM results we develop the qMS-Q FF to enable the use of MD for determining the equation-of-state as a function of $T$ and $P$. Also using MD we extracted thermodynamic data to determine the solid-solid and solid-liquid phase transitions as a function of temperature. This procedure is fully first principles with no use of experimental data. In this paper we apply this procedure to the $\mathrm{B} 1(\mathrm{NaCl})$ and $\mathrm{B} 2(\mathrm{CsCl})$ phases of $\mathrm{MgO}$, and we are in the process of doing similar calculations on other systems.

These results show a pressure-induced phase transition from the high-density phase (B1) to the low-density phase (B2) at a pressure of $P \sim 400 \mathrm{GPa}$. This agrees with recent simulations ${ }^{6}$ where the $\mathrm{B} 1-\mathrm{B} 2$ phase transition at $T=0 \mathrm{~K}$ was found to be $450 \mathrm{GPa}$. This is consistent with the experimental observation that no transition occurs below $227 \mathrm{GPa}$. These results were used to calculate the phase diagram of $\mathrm{MgO}$ for pressures up to $500 \mathrm{GPa}$ and temperatures up to $8000 \mathrm{~K}$, providing, we believe, the first report of finite temperature B1-B2 phase transition. The melting temperature at zero pressure is in very good agreement with experiment. For high pressures our melting temperature is higher than the experimental one.

This phase diagram shows that the $\mathrm{B} 1$ structure of $\mathrm{MgO}$ is stable up to $\sim 300 \mathrm{GPa}$ (at $T 7000 \mathrm{~K}$ ). Since the maximum pressure in the lower mantle of the earth is $\sim 140 \mathrm{GPa}$, no transformation to $\mathrm{B} 2$ is expected.

\section{ACKNOWLEDGMENTS}

We thank Dr. Ersan Demiralp for useful discussions. This research was funded by a grant from DOE-ASCI. A.S. acknowledges financial support from FOMEC (Argentina). The facilities of the MSC are also supported by grants from NSF (CHE 95-22179), ARO (MURI), ARO (DURIP), BP Chemical, Exxon, Dow Chemical, Owens-Corning, Avery Dennison, Chevron Petroleum Technology Co., Asahi Chemical, Chevron Chemical Co., Chevron Research Technology Co., and Beckman Institute. 
${ }^{1}$ T. S. Duffy, R. J. Hemley, and H.-k. Mao, Phys. Rev. Lett. 74, 1371 (1995).

${ }^{2}$ K. J. Chang and M. L. Cohen, Phys. Rev. B 30, 4774 (1984).

${ }^{3}$ M. S. T. Bukowinski, Geophys. Res. Lett. 12, 536 (1985).

${ }^{4}$ M. J. Mehl, R. E. Cohen, and H. Krakauer, J. Geophys. Res. 93, 8009 (1988).

${ }^{5}$ Donald G. Isaak, Ronald R. Cohen, and Michael J. Mehl, J. Geophys. Res. 95, 7055 (1990).

${ }^{6}$ B. B. Karki, L. Stixude, S. J. Clark, M. C. Warren, G. J. Ackland, and J. Crain, Am. Mineral. 82, 51 (1997).

${ }^{7}$ L. Vocadlo and G. D. Price, Phys. Chem. Miner. 23, 42 (1997).

${ }^{8}$ R. E. Cohen, in Physics Meets Mineralogy, edited by H. Aoki, Y. Syono, and R. J. Hemley (Cambridge University Press, Cambridge, England, in press); R. E. Cohen and J. Weitz, in High Pressure-Temperature Research: Properties of Earth and Planetary Materials, edited by M. H. Manghnani and T. Yagi (AGU, Washington, D.C., 1998), pp. 185-196.

${ }^{9}$ A. B. Belonoshko and L. S. Dubrovinski, Am. Mineral. 81, 303 (1996).

${ }^{10}$ A. Zerr and R. Boehler, Nature (London) 371, 506 (1994).

${ }^{11}$ Ronald E. Cohen and Z. Gong, Phys. Rev. B 50, 12301 (1994).

${ }^{12}$ Finite Size Scaling and Numerical Simulations in Statistical Systems, edited by V. Privman (World Scientific, Singapore, 1990).

${ }^{13}$ E. Demiralp, T. Çăgin, and W. A. Goddard III, Phys. Rev. Lett. 82, 1708 (1999).

${ }^{14}$ P. Hohenberg and W. Kohn, Phys. Rev. 136, 864B (1964).

${ }^{15}$ W. Kohn and L. J. Sham, Phys. Rev. 140, 1133A (1965).

${ }^{16}$ M. C. Payne, M. P. Teter, D. C. Allan, T. A. Arias, and J. D. Joannopoulos, Rev. Mod. Phys. 64, 1045 (1992).
${ }^{17}$ J. P. Perdew and Y. Wang, Phys. Rev. B 46, 6671 (1992); J. A. White and D. M. Bird, ibid. 50, 4954 (1994).

${ }^{18}$ See our web site (http://www.wag.caltech.edu/publications/ papers/). In addition, see EPAPS Document No. E-PRBMDO-60-023946 for supplementary tables. This document may be retrieved via the EPAPS homepage (http:// www.aip.org/pubservs/epaps.html) or from ftp.aip.org in the directory /epaps/. See the EPAPS homepage for more information.

${ }^{19}$ I. Jackson and H. Niesler, in High Pressure Research in Geophysics, edited by S. Akimoto and M. H. Manghnani (Center for Academic Publishing, Tokyo, 1982), p. 93.

${ }^{20}$ H. K. Mao and P. M. Bell, J. Geophys. Res. 84, 4533 (1979).

${ }^{21}$ T. Çağin, E. Demiralp, and W. A. Goddard III, in Microscopic Simulations of Interfacial Phenomena in Solids and Liquids, edited by S. Phillpot, P. Bristowe, D. Stroud, and J. Smith (Materials Research Society, Warrendale, PA, 1998), Vol. 492, p. 287.

${ }^{22}$ E. Demiralp, T. Çağin, N. T. Huff, and W. A. Goddard III, in Proceedings of the XVIII International Congress on Glass, 1998, edited by M. K. Choudhary, N. T. Huff, and C. H. Drummond (American Ceramic Society, Westerville, OH, 1998); N. T. Huff, E. Demiralp, T. Çağin, and W. A. Goddard III, J. Noncryst. Solids 253. 133 (1999).

${ }^{23}$ A. Rappé and W. A. Goddard III, J. Phys. Chem. 95, 3358 (1991).

${ }^{24}$ N. Karasawa and W. A. Goddard III, J. Phys. Chem. 93, 7320 (1989).

${ }^{25}$ Iris Inbar and R. E. Cohen, Geophys. Res. Lett. 22, 1533 (1995).

${ }^{26}$ M. Parinello and A. Rahman, J. Appl. Phys. 52, 7182 (1981).

${ }^{27}$ D. Frenkel and B. Smit, Understanding Molecular Simulation, from Algorithms to Applications (Academic, San Diego, 1996). 